\title{
Chronic Pericardial Constriction Induced Severe Ischemic Hepatitis Manifesting as Hypoglycemic Attack
}

\author{
Tetsuya Nomura, MD; Natsuya Keira, MD; Yota Urakabe, MD; Daisuke Naito, MD; \\ Mayuka Nakayama, MD; Atsumichi Kido, MD; Hidetoshi Kanemasa, MD*; \\ Hiroaki Matsubara, MD**; Tetsuya Tatsumi, MD
}

\begin{abstract}
Ischemic hepatitis, otherwise known as "shock liver", is characterized by a massive, but transient increase in serum transaminase levels, usually associated with cardiac failure. A patient who did not have a predisposition to hypoglycemia was discovered at home with disturbed consciousness caused by hypoglycemia. She had been diagnosed as having constrictive pericarditis several years earlier and had developed ischemic hepatitis. Though the high serum transaminase levels were rapidly normalized, severe jaundice gradually developed and the patient finally died of multiple organ failure. Hypoglycemia, which is considered secondary to reduced gluconeogenesis in the exhausted liver, is a rare complication of constrictive pericarditis. (Circ J 2009; 73: 183-186)
\end{abstract}

Key Words: Constrictive pericarditis; Heart failure; Hypoglycemia; Ischemic hepatitis

$\mathbf{T}$

he correlation between cardiac disease and liver dysfunction is well documented, but constrictive pericarditis $(\mathrm{CP})$ rarely presents with ischemic hepatitis.

\section{Case Report}

A 57-year-old woman with disturbed consciousness was transported to the emergency room. Her blood pressure was $104 / 88 \mathrm{mmHg}$, pulse rate was $98 / \mathrm{min}$ with regular rhythm, and she was afebrile. Her jugular vein was distended and showed venous collapse in the diastolic phase. Her legs were considerably edematous. A grade $2 / 6$ systolic regurgitant murmur was audible at the right sternal border in the $4^{\text {th }}$ intercostal space, but excess heart sounds were unclear. On pulmonary auscultation, sounds were hardly heard in the left lung field. Computed tomography (CT) did not show any evidence of organic lesion in the brain. Though she had no predisposition to hypoglycemia, her serum glucose level was $23 \mathrm{ng} / \mathrm{ml}$ and a venous infusion of glucose restored normal consciousness. The first blood gas analysis under $5 \mathrm{~L} / \mathrm{min}$ oxygen inhaled when just arriving at the hospital showed severe respiratory acidosis (Table 1). However, after recovery of consciousness, the hypercapnia was soon reversed. In addition to low levels of serum glucose, laboratory results showed high necro-inflammatory activity of the liver with an alanine transferase (ALT) level of $1,755 \mathrm{U} / \mathrm{L}$, aspartate transferase (AST) level of 3,615 U/L, lactate dehydrogenase (LDH) level of 5,160 U/L, 47\% prothrombin time ratio, and total bilirubin (T-Bil) level of $3.9 \mathrm{mg} / \mathrm{dl}$

(Received October 29, 2007; revised manuscript received March 6, 2008; accepted April 3, 2008; released online November 11, 2008)

Department of Cardiovascular Medicine, *Department of Gastroenterology, Nantan General Hospital, Nantan and **Department of Cardiovascular Medicine, Kyoto Prefectural University School of Medicine, Kyoto, Japan

Mailing address: Tetsuya Nomura, MD, Department of Cardiovascular Medicine, Nantan General Hospital, 25 Yagi-Ueno, Yagi-cho, Nantan 629-0197, Japan. E-mail: t2-ya@za2.so-net.ne.jp

All rights are reserved to the Japanese Circulation Society. For permissions, please e-mail: cj@j-circ.or.jp
Table 1 Laboratory Data on Admission

\begin{tabular}{|c|c|}
\hline$W B C$ & $6,730 / \mu l$ \\
\hline$R B C$ & $3.82 \times 10^{6} / \mu l$ \\
\hline$H b$ & $10.9 \mathrm{~g} / \mathrm{dl}$ \\
\hline Hct & $36.2 \%$ \\
\hline Plt & $162 \times 10^{3} / \mu l$ \\
\hline$B U N$ & $32.4 \mathrm{mg} / \mathrm{dl}$ \\
\hline Cre & $1.53 \mathrm{mg} / \mathrm{dl}$ \\
\hline $\mathrm{Na}$ & $126 \mathrm{mmol} / \mathrm{L}$ \\
\hline$K$ & $5.1 \mathrm{mmol} / \mathrm{L}$ \\
\hline $\mathrm{Cl}$ & $86 \mathrm{mmol} / \mathrm{L}$ \\
\hline Amylase & $50 I U / L$ \\
\hline$C K$ & $362 U / L$ \\
\hline$A S T$ & 3,615 U/L \\
\hline$A L T$ & $1,755 \mathrm{U} / \mathrm{L}$ \\
\hline$L D H$ & $5,160 \mathrm{U} / \mathrm{L}$ \\
\hline$A L P$ & $169 \mathrm{U} / \mathrm{L}$ \\
\hline$T$-Bil & $3.9 \mathrm{mg} / \mathrm{dl}$ \\
\hline$\gamma-G T$ & $52 U / L$ \\
\hline$C R P$ & $1.9 \mathrm{mg} / \mathrm{dl}$ \\
\hline$B S$ & $23 \mathrm{mg} / \mathrm{dl}$ \\
\hline $\mathrm{NH}_{3}$ & $79 \mu \mathrm{g} / \mathrm{dl}$ \\
\hline$B N P$ & $432 \mathrm{pg} / \mathrm{ml}$ \\
\hline \multicolumn{2}{|l|}{$\left(5 \mathrm{~L} / \mathrm{min} \mathrm{O}_{2}\right.$ inhaled $)$} \\
\hline$p H$ & 7.188 \\
\hline $\mathrm{PCO}_{2}$ & $84.2 \mathrm{mmHg}$ \\
\hline $\mathrm{PO}_{2}$ & $378.8 \mathrm{mmHg}$ \\
\hline $\mathrm{HCO}_{3}$ & $31.3 \mathrm{mmol} / \mathrm{L}$ \\
\hline$B E$ & $0.2 \mathrm{mmol} / \mathrm{L}$ \\
\hline$U-p H$ & 5.5 \\
\hline$U$-protein & $1+$ \\
\hline U-glucose & - \\
\hline U-ketones & - \\
\hline U-occult blood & $3+$ \\
\hline$U$-urobilinogen & $1+$ \\
\hline$U$-bilirubin & - \\
\hline
\end{tabular}

$\overline{A S T \text {, aspartate transferase; } A L T \text {, alanine transferase; } L D H \text {, lactate }}$ dehydrogenase; T-Bil, total bilirubin.

(Table 1). She was seronegative for both hepatitis B virus (HBV) and hepatitis $\mathrm{C}$ virus (HCV), and had not taken any hepatotoxic medicines. Ultrasonography showed no findings of the abdominal organs but there was a small amount of ascetic fluid. Her recent laboratory data and physical 
$\mathbf{A}$



\section{B}

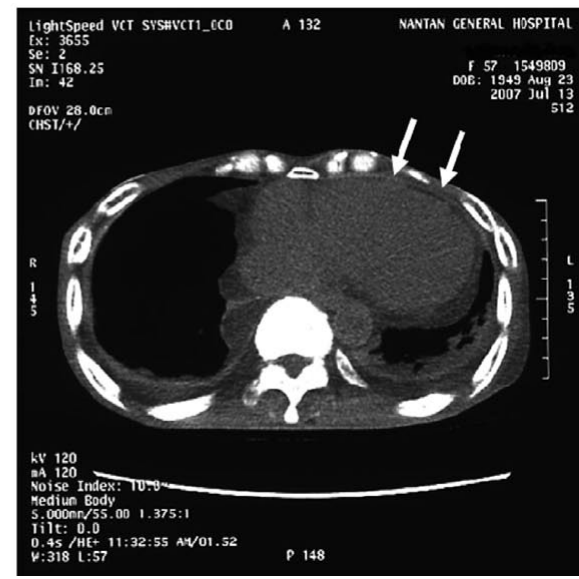

$\mathbf{A}$

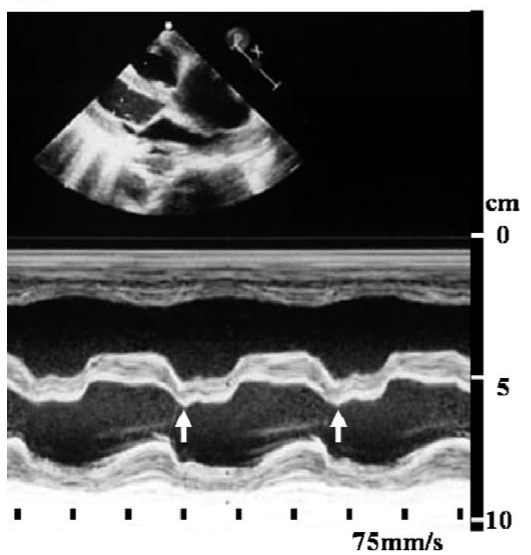

B

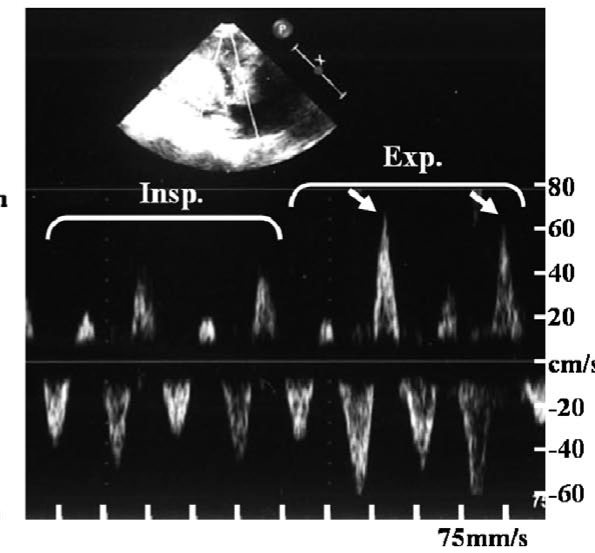

Fig 1. Chest radiogram (A) and chest computed tomography (CT, B) on admission. CT scan shows partially thickened pericardium (arrows).
Fig 2. (A) Parasternal long-axis M-mode echocardiogram demonstrating typical septal notch in the early diastolic phase (arrows). (B) Doppler flow velocity of hepatic vein showing expiratory increase in diastolic flow reversal (arrows). findings did not indicate that she was in overt liver cirrhosis. She was hospitalized for thorough examination and intensive medical treatment of her general condition.

She had a history of breast cancer and had undergone mammotomy 7 years earlier. Approximately 1 year later she developed massive pericardial and left pleural bloody effusion. Though cytological examination of the effusion did not show definitive evidence of malignancy, her medical history strongly suggested that it derived from the malignancy. She underwent left lung pleurodesis and cisplatin infusion into the pericardial cavity. Thereafter, she developed pretibial edema and general malaise, which were thought to be related to chronic right heart failure because of pericardial constriction. Therefore, she was prescribed medication for heart failure.

Chest radiography showed an enlarged cardiothoracic ratio and reduced amount of left lung (Fig 1A). Chest CT demonstrated a small amount of bilateral pleural and pericardial effusion and a partially thickened pericardium, but there was no obvious calcification or sclerotic changes of the pericardium (Fig 1B). A 12-lead electrocardiogram showed 104 beats/min sinus rhythm with pulmonary P wave and non-specific ST-T changes. Echocardiography with Doppler examination was performed in detail. Normal ventricular systolic function (31\% fractional shortening) with enlargement of the atrium, inferior vena cava, and hepatic veins was observed. An early diastolic septal notch was shown by M-mode (Fig 2A). Severe tricuspid regurgitation of $3.56 \mathrm{~m} / \mathrm{s}$ at the peak velocity was recorded, which suggested right ventricle (RV) systolic pressure was over $60 \mathrm{mmHg}$. Doppler assessment of the hepatic vein demonstrated biphasic forward flow followed by flow reversal on atrial contraction. There was an expiratory increase in the diastolic flow reversal (47.9\%) (Fig 2B). Mitral and tricuspid Doppler inflow patterns were measured; the transmitral flow velocity showed a restrictive pattern (E/A 0.62, deceleration time $135 \mathrm{~ms}$ ) with a typical inspiratory decrease in E wave $(27.0 \%)$. Opposite changes were noted in the tricuspid flow velocity as the E wave increased with inspiration $(46.1 \%)$. Based on these findings, the final diagnosis was $\mathrm{CP}$, causing ischemic hepatitis.

To treat right heart predominant failure, we used lowdose dobutamine and milrinone together, in addition to carperitide. For some time after admission, the patient's urinary outflow was sufficient to improve the congestion, as assessed by physical examination and echocardiography. The elevated levels of the aminotransferases gradually normalized, but the blood glucose and ammonia levels continued to fluctuate, causing occasional episodes of unconsciousness (Fig 3). Over time, the inotropic agents became less effective and there was reduced urinary volume and respiratory impairment. At the same time, the total bilirubin level was also increasing and signs of multiple organ failure emerged. The patient died on day 18 of hospitalization (Fig 3). 


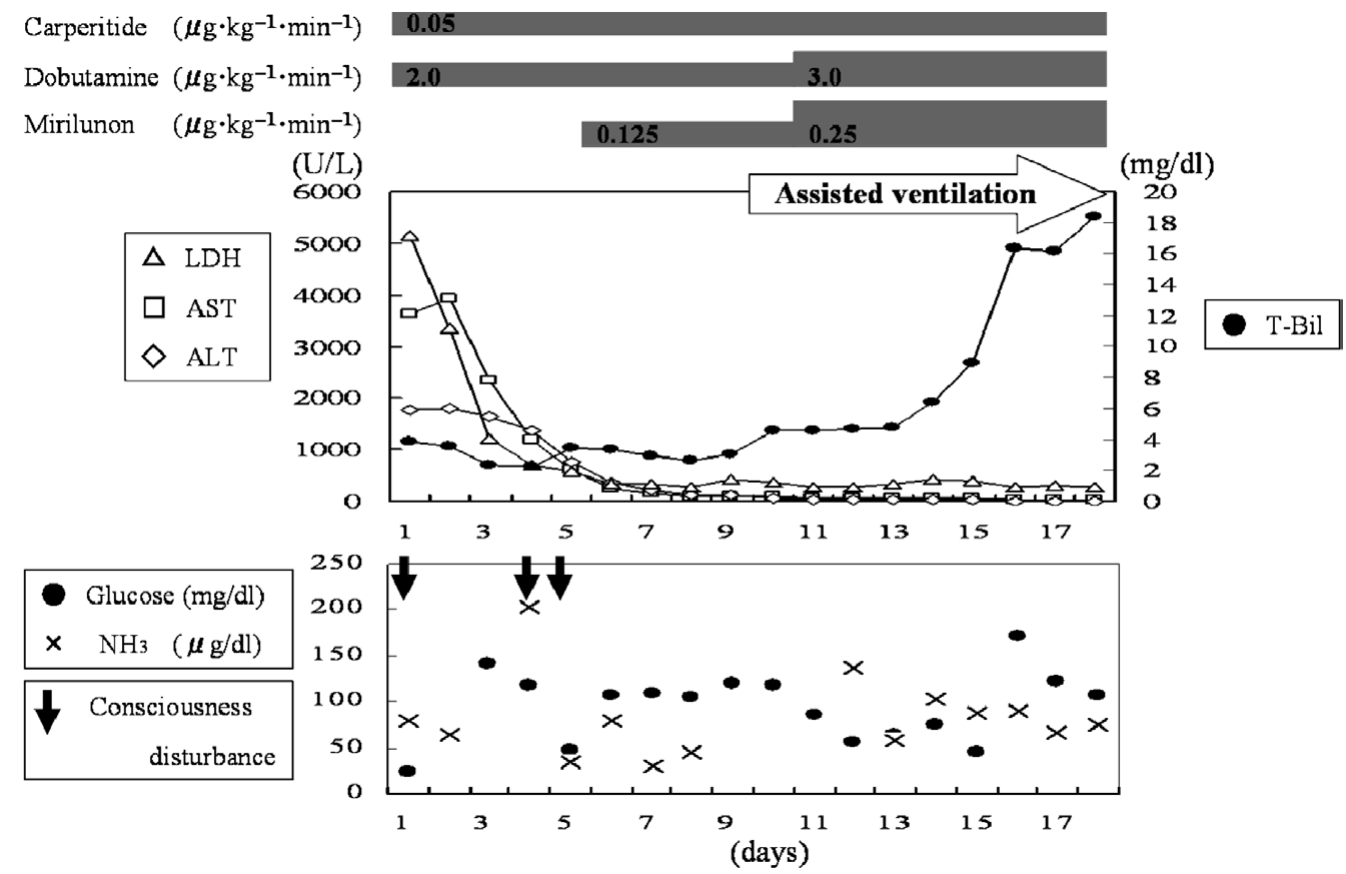

Fig 3. Changes in the medical treatment and serum markers during hospitalization. ALT, alanine transferase; AST, aspartate transferase; $\mathrm{LDH}$, lactate dehydrogenase; T-Bil, total bilirubin.

\section{Discussion}

Although approximately 30-50\% of cases of cardiac failure are also accompanied by slight jaundice and increased amminotransferase levels, mostly AST than ALT, the levels in most patients are no more than twice the normal limit. However, we sometimes encounter the clinical entity that is commonly referred to as shock liver or ischemic hepatitis, which is characterized by transaminase elevations greater than 20 times the normal level, but which rapidly return to near normal levels, because of inadequate oxygen uptake by the centrilobular hepatocytes resulting in necrosis. The most common cause is insufficient hepatic perfusion in the setting of passive liver congestion or chronic liver disease. The vast majority of patients with ischemic hepatitis have severe underlying cardiac disease, mostly right-sided heart failure, and high central venous pressure that had often leads to passive congestion of the liver!

Henrion et al reported that the occurrence of ischemic hepatitis caused by congestive heart failure is triggered by one or more acute events, most often of cardiogenic origin? The most frequent acute events are cardiac arrhythmia $(52.5 \%)$, acute pulmonary edema (34\%), and acute myocardial infarction (12.5\%). Although CP is characterized by considerable right-sided heart failure, only a few reports describe liver dysfunction related to it 3,4 The disturbance of consciousness accompanying this pathophysiology is considered to be related to hepatic encephalopathy, and there is no report of hypoglycemia-induced loss of consciousness caused by $\mathrm{CP}$.

In the case of CP, pulmonary artery and RV systolic pressures are usually moderately elevated, in the $35-45 \mathrm{mmHg}$ range. Pulmonary hypertension, such as the present case, is not a feature of $\mathrm{CP}$ and indicates coexisting cardiac or pulmonary disease. We speculate that the clinical background of this patient (ie, postoperative pericarditis and pleurode- sis) contributed in some degree to the development of pulmonary hypertension.

The consciousness disturbance and hypercapnia on admission and the echocardiographic findings of pulmonary hypertension forced us to differentiate pulmonary embolism (PE). The clinical decision scoring system is reliable for stratifying patients into high clinical probability or non-high clinical probability of PE5 The present patient scored only 1.5 (HR $>100$ beats/min), which categorized her as "PE unlikely".

$\mathrm{CP}$ is a disorder with various causes such as idiopathic origin, viral infection, tuberculosis, cardiovascular surgery, radiation therapy, trauma and malignant invasion. It is characterized by 2 hemodynamic states: exaggerated ventricular interdependence and dissociation of intracardiac and intrathoracic pressures? Traditionally, the presence of increased pericardial thickness is considered the essential diagnostic feature of $\mathrm{CP}$, and $\mathrm{CT}$ and magnetic resonance imaging are useful for detecting it? However, Talreja et al demonstrated cases of $\mathrm{CP}$ with histologically normal pericardium, and they emphasized the importance of assessing the hemodynamic data in making the diagnosis of constrictive physiology? Increased pericardial thickness does not necessarily mean constriction, but normal pericardial thickness does not necessarily exclude a diagnosis of CP.

Our patient had a history of bloody pericardial effusion thought to be derived from metastasis of breast cancer. Since that episode, we observed progression of the hemodynamic state manifesting as $\mathrm{CP}$, even though there was not any conspicuous effusion. Malignant tumor of the heart (malignant lymphoma, angiosarcoma, mesothelioma, breast cancer or lung cancer), irrespective of the primary or metastatic tumor, is commonly invasive to the pericardium or myocardium, which often results in diastolic dysfunction and some of these cases present with $\mathrm{CP} 9,10$

Radical pericardiectomy is the definitive treatment for constriction and it is emphasized that timely surgery is 
needed!1,12 However, the perioperative mortality is relatively high, especially in cases showing severe impairment of visceral pericardium, severe liver dysfunction, intense calcification of the pericardium and complicated myocardial damage. Persistent impairment of her general condition in this case, including respiratory and neurological disorders, hampered the decision to perform surgery.

During the clinical course, the congestion caused by heart failure gradually improved and the AST, ALT and LDH levels rapidly normalized. However, the T-Bil level gradually increased, reaching a peak of $18.4 \mathrm{mg} / \mathrm{dl}$, and severe jaundice developed. When such severe jaundice occurs, fulminant hepatic failure becomes fatal ${ }^{3}$

We present a case of ischemic hepatitis caused by chronic CP. During progression of the illness, the rare pathological changes of hypoglycemia and severe jaundice developed. Approximately 6 years passed since the onset of the constrictive state was recognized and we should have determined a time for pericardiectomy before the illness progressed.

\section{References}

1. Seeto RK, Fenn B, Rockey DC. Ischemic hepatitis: Clinical presentation and pathogenesis. Am J Med 2000; 109: 109-113.

2. Henrion J, Schapira M, Luwaert R, Colin L, Delannoy A, Heller FR. Hypoxic hepatitis: Clinical and hemodynamic study in 142 consecutive cases. Medicine 2003; 82: 392-406.

3. Kerzner R, Barzilai B, Satyanarayana R. Constrictive pericarditis and hepatic encephalopathy. Lancet 2002; 359: 1895.
4. Arora A, Seth S, Acharya SK, Sharma MP. Hepatic coma as a presenting feature of constrictive pericarditis. Am J Gastroenterol 1993; 88: $430-432$.

5. Wells PS, Ginsberg JS, Anderson DR, Kearon C, Gent M, Turpie AG, et al. Use of a clinical model for safe management of patients with suspected pulmonary embolism. Ann Intern Med 1998; 129: $997-$ 1005.

6. Myers RBH, Spodick DH. Constrictive pericarditis: Clinical and pathophysiologic characteristics. Am Heart J 1999; 138: 219-232.

7. Dato I, Coluzzi G, Al-Mohanni G, Della Bona R, Piro M, Natale L, et al. A young man with intractable ascites and effort dyspnoea without echocardiographic signs of pericardial thickening: The importance of clinical investigation, CT scan and MRI in the diagnosis of constrictive pericarditis. Int J Cardiol 2008; 128: e79-e81.

8. Talreja DR, Edwards WD, Danielson GK, Schaff HV, Tajik AJ, Tazelaar HD, et al. Constrictive pericarditis in 26 patients with histologically normal pericardial thickness. Circulation 2003; 108: $1852-1857$

9. Watanabe A, Sakata J, Kawamura H, Yamada O, Matsuyama T. Primary pericardial mesothelioma presenting as constrictive pericarditis. Jpn Circ J 2000; 64: 385-388.

10. Karadede A, Ülgen MS, Temamogullari AV, Toprak N. A complicated case of pericardial hydatid cyst manifesting as constrictive pericarditis. Can J Cardiol 2000; 16: 673-676.

11. Senni M, Redfield MM, Ling LH, Danielson GK, Tajik AJ, Oh JK. Left ventricular systolic and diastolic function after pericardiectomy in patients with constrictive pericarditis. J Am Coll Cardiol 1999; 33: $1182-1188$

12. Güneri S, Nazli C, Kinay O, Kirimli O, Mermut C, Hazan E. Chylous ascites due to constrictive pericarditis. Int J Card Imaging 2000; 16: 49-54.

13. Kaymakcalan H, Dourdourekas D, Szanto PB, Steigmann F. Congestive heart failure as cause of fulminant hepatic failure. Am J Med 1978; 36: 384-388. 